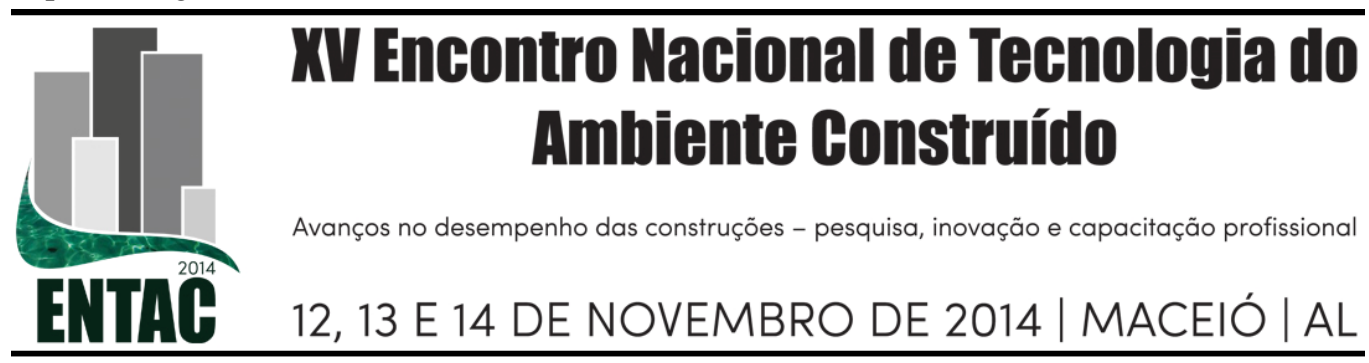

\title{
PRÁTICAS DE GERENCIAMENTO ENXUTO EM ESCRITÓRIOS DE PROJETO DE GOIÂNIA
}

\section{SANTOS, Vinícius Carrião dos (1); AMARAL, Tatiana Gondim do (2); BATISTA, Danilo Gonçalves (3)}

(1) Universidade Federal de Goiás, (62) 81830187, e-mail: vinicius.arquiteto@yahoo.com.br (2) Universidade Federal de Goiás, e-mail: tatiana_amaral@hotmail.com, (3) Universidade Federal de Goiás, e-mail:

danilogancalves@gmail.com

\begin{abstract}
RESUMO
Com foco no processo de projeto de edifícios este trabalho investigou a existência de práticas de gestão coerentes com o lean design management (gerenciamento de projeto enxuto) com vistas à redução das deficiências do gerenciamento do processo de projeto. A fundamentação teórica sobre o lean design management é recente e o tema tem adquirido importância por sua potencial contribuição ao processo de projeto de edifícios por meio de ferramentas simples e, sobretudo, pela ênfase no comprometimento dos agentes do processo. A pesquisa se classifica como estudo de caso, desenvolvido em dois escritórios de projeto de edifícios de multipavimentos da cidade de Goiânia. Os resultados demonstraram o estágio ainda preliminar das ações para melhoramento do processo de projeto e as dificuldades enfrentadas. A pequena amostragem do estudo não permitiu o aprofundamento das causas destas dificuldades, nem o estabelecimento do nível de compreensão de conceitos e ferramentas enxutas por parte dos escritórios. A partir dos tópicos criados para a análise do processo de projeto pôde-se definir qual deles é preferencial na alocação de recursos para melhorias incrementais no processo. Também foi possível visualizar quesitos de gestão do processo que parecem variar com a disciplina de projeto. Este estudo foi útil para destacar que há um interesse dos escritórios de projeto em melhorar seus processos de trabalho e de que novos estudos acadêmicos possam auxiliá-los a superar as dificuldades percebidas e que se mostram de difícil transposição.
\end{abstract}

Palavras-chave: Lean design management; gerenciamento de projetos; processo de projeto de edifícios.

\begin{abstract}
Focusing on the building design process, this paper studied the occurrence of management practices aligned with the lean design management with the aim to reduce the management deficiencies in the design process. The theoretical foundation of the lean design management is recent and it has gained importance in literature for its potential contribution to the building design process through simple tools and, especially, by emphasizing the commitment of the process agents. Two design firms of multiple floor buildings of the city of Goiania were investigated through semi-structured interviews. The results demonstrated the preliminary stage of further actions to improve the design process and the difficulties faced. The small sampling of the study did not allow the deepening of the difficulties causes, nor establish the level of understanding of lean tools and concepts by the firms. From the topics created for the analysis of the design process could be defined which one is preferred in allocating resources to generate process improvements. It was also possible to point questions of design process management that seem to vary with the design discipline. This study was useful to highlight that there is an interest by the design firms in improve their work processes and that new academic researches help them overcome the difficulties.
\end{abstract}

Keywords: Lean design management; project management; building design process. 


\section{INTRODUÇÃO}

Do ponto de vista do gerenciamento, o processo de projeto de edifícios é caracterizado como ineficiente, no qual se podem identificar vários tipos de perdas (TILLEY, 2005; KOSKELA et $a l .$, 1997). Pesquisas fundamentadas no pensamento enxuto têm sido desenvolvidas com o intuito de identificar e desenvolver formas e ferramentas para que o processo de projeto tornese eficiente e alcance o valor desejado pelo cliente (HANSEN; OLSSON, 2011; El.REIFI; EMMITT, 2011; EMMITT et al., 2011; SMITH et al., 2011).

O processo de projeto é complexo e envolve fluxos de características distintas assim como a atuação de diversos agentes com diferentes atribuições. Por isso, os trabalhos citados anteriormente enfocam agentes diferentes do processo, mas com um só objetivo.

De acordo com Emmitt et al. (2011) a maioria das pesquisas relacionadas ao tema diz respeito a projetos de construção muito grandes ou pertencentes a grandes organizações contratantes, tendo-se na literatura uma pequena proporção de trabalhos dedicados às pequenas e médias empresas, que representa grande parcela das empresas do setor da construção.

$\mathrm{O}$ artigo insere-se no universo de pesquisas na área de gestão que evidenciam as dificuldades e deficiências assim como a importância da fase de projeto. Muitos são os problemas identificados tais como a deficiência das informações, as falhas de comunicação, as perdas e dificuldades como a falta de entrosamento entre os técnicos envolvidos, a maior complexidade das edificações contemporâneas que exigem mais especialistas, o aumento da demanda produtiva e da exigência por qualidade e desempenhos compatíveis, o trabalho à distância e com equipes multinacionais, entre outros.

\section{OBJETIVOS}

Explorar as estratégias de ação enxutas para a redução das deficiências do gerenciamento do processo de projeto em escritórios de projeto de edifícios de multipavimento goianienses.

\section{OS PROBLEMAS DE EFICIÊNCIA DO PROJETO E SUA GESTÃO}

Atrasos, desentendimentos e incertezas caracterizam o processo de projeto convencional enquanto a documentação deficiente e a baixa qualidade marcam o produto de projeto. Como consequências para o processo temos os retrabalhados e outros tipos de perdas, já o produto construído fica aquém dos requisitos desejados pelo cliente (SMITH et al., 2011).

O uso de informações incompletas, a tomada de decisão vacilante, a locação inadequada de recursos, a falta de coordenação, a desconsideração de decisões anteriores em etapas avançadas do projeto e a comunicação deficiente são falhas de gerenciamento que prejudicam o processo de projeto e que ainda persistem em graus variáveis apesar da evolução da prática da gestão (KOSKELA et al., 1997; TILLEY, 2005; FORMOSO \& TZORTZOPOULOS, 1999).

Tilley (2005) aponta a deficiência do programa de necessidades (briefing) e as falhas de detalhamento como os principais fatores para a baixa construtibilidade, a má documentação de projeto e a falta de atendimento aos requisitos do cliente. Situação que se acentua com o atual quadro de queda do preço da elaboração de projeto, prazos menores para a conclusão dos planos e o aumento da complexidade dos edifícios.

Três são os focos para a atuação sobre os processos de projeto com vistas a seu aprimoramento. As falhas de gerenciamento citadas acima evidenciam que o fluxo de informação e o planejamento do processo de projeto são determinantes. Por outro lado, apenas a redução dos desperdícios no processo não torna um projeto bem sucedido se ele não atender 
os requisitos do cliente, isto é, o gerenciamento do valor (KOSKELA et al.,1997; HANSEN; OLSSON, 2011; EL.REIFI; EMMITT, 2011).

\subsection{O fluxo de informações}

O fluxo de informações pode ser analisado a partir de três critérios: os tempos de ciclo, os trabalhos em aberto e as restrições ou gargalos do fluxo (TRIBELSKY; SACKS, 2010). Estes critérios tendem a ser cada vez mais automatizados com os avanços das ferramentas de modelagem da informação no processo de projeto (Building Informatiom Modelling - BIM) em substituição às ferramentas tradicionais de desenho assistido por computador.

Tempo e informação são fatores críticos na construção. Tribelsky e Sacks (2010) investigaram o gerenciamento do fluxo da informação e identificaram não só a falta de indicadores para avaliar o fluxo de informações, que denuncia que o processo não é conhecido a fundo, assim como a falta de medidas ou soluções gerenciais que objetivem aprimorar o gerenciamento e o conteúdo das informações compartilhadas. Os autores estudaram também os intervalos e os meios utilizados para a troca das informações, se via correio eletrônico, CDs ou extranets, e ainda o tamanho, o conteúdo e à qualidade das informações.

Verificou-se que o volume de informações compartilhadas de uma só vez é grande e isso dificulta a rápida compreensão de quem o recebe, de modo que conteúdos importantes podem passar despercebidos. Contribuem também para a dispersão do conteúdo os longos intervalos de compartilhamento e as repetições.

\subsection{O planejamento do processo de projeto}

Ferramentas de controle e o comprometimento da equipe de trabalho contribuem para a redução das deficiências no planejamento do processo de projeto. Koskela et al. (1997), Tilley (2005) e Emmitt et al. (2011) apontam que o comprometimento de todos os envolvidos no empreendimento é determinante para a compreensão dos meandros do processo e o estabelecimento das promessas que regem os compromissos estabelecidos. Sem estes compromissos, ferramentas de planejamento e controle, como Last Planner, Design Structure Matrix (DSM), check-lists, briefs, set-base design e cronogramas pouco contribuirão para o atendimento das metas de prazo e de qualidade dos produtos (TILLEY, 2005).

Koskela et al. (1997), Tilley (2005), Tribelsky; Sacks (2010) e El.Reifi; Emmitt (2011) destacam que o projeto é gerenciado e entendido a partir de princípios obsoletos, pouco adequados ao caráter iterativo da projetação. Por isso, a liderança deve existir como recurso para tornar o processo eficiente e efetivo.

A desconsideração da interrelação entre as tarefas planejadas e a falta de organização do fluxo de informações são os responsáveis pelo atraso na obtenção dos dados para o projeto, pela deficiência das informações e pelo retardo na conclusão das etapas de projeto (TILLEY, 2005).

\subsection{0 gerenciamento do valor}

O atendimento aos requisitos do cliente é o foco da elaboração do produto e é estratégico para o projetista se manter no mercado. Valor pode ser subjetivo ou objetivo e extrapola as medidas financeiras. Geralmente varia com o tempo e conforme o contexto, mas é avaliável e apreciável (THYSSEN et al., 2010).

O gerenciamento do valor favorece uma melhor documentação do processo, contribui para a redução de suas incertezas e orienta a inovação (TILLEY, 2005). Portanto, o comprometimento da equipe envolvida associado a ferramentas de seleção de soluções de 
projeto como a quality deployment function (QDF) é determinante, tal como acontece com o planejamento do processo de projeto (KOSKELA et al. 1997; EMMITT et al., 2011).

No gerenciamento do valor o elemento primordial é o programa de necessidades (briefing), que pode ser único e definido no início do projeto, reunindo o máximo de informações possíveis, ou dinâmico a ser trabalhado ou completado e revisado a cada etapa do projeto de modo a proteger o processo de elaboração de suposições excessivas e às vezes necessárias para que o projeto não pare. Estas suposições surgem por falta de tomada de decisão ou falta de informação no momento adequado e caracterizam circunstância corriqueira na prática convencional de gerenciamento (KOSKELA et al. 1997; TILLEY, 2005; EMMITT et al., 2011).

\section{LEAN DESIGN MANAGEMENT (LDM)}

A gestão enxuta (lean) aplicada ao desenvolvimento de projetos proporciona uma produção mais ágil, com melhor aproveitamento da dedicação profissional e a atenção adequada aos requisitos do cliente. O LDM associa recursos da construção enxuta e das lições e ferramentas do gerenciamento de projetos. Constitui uma abordagem que pretende aumentar a eficiência e a qualidade dos resultados do projeto, maximizando o alcance do valor para os clientes do processo (TILLEY, 2005; EGAN, 1998 apud EL.REIFI; EMMITT, 2011).

No gerenciamento de projetos de construção ainda persiste a compreensão do processo como uma sequência de atividades, como ocorre na etapa de obra. Esta associação durante a fase de projetos só é aplicável a partir da etapa de detalhamento (TRIBELSKY; SACKS, 2010). Diferentemente, as etapas iniciais do projeto não são plenamente lineares e ciclos podem existir como momentos para análise e seleção de alternativas. Nesta fase criativa, o tempo é dificilmente determinado e por isso ferramentas tradicionais de programação como o Critical Path Method (CPM) são inadequadas (EL.REIFI; EMMITT, 2011).

O Integrated Project Delivery (IPD) reúne conceitos e princípios da produção enxuta, técnicas e recursos informacionais (BIM), ferramentas gerenciais e conceitos de relação interpessoal, como a confiança e a integração, para criar um ambiente produtivo que favoreça maior produtividade e eficiência com menos desperdício e maior valor para o cliente (AIA, 2007). O IPD, considerado como um produto do LDM, é um processo de produção de edifícios que procura integrar pessoas, sistemas, negócios e recursos num trabalho colaborativo que busca minimizar as perdas, otimizar a eficiência desde a concepção do empreendimento até a entrega e avaliação pelo usuário.

No entanto, a aplicação de práticas enxutas exige dedicação e consome recursos. Seus resultados não se estabelecem em curto prazo e o conjunto de soluções LDM é, em geral, pouco generalizável. Em função destas características, as práticas LDM são consideradas desinteressantes para muitas empresas (El.REIFI; EMMITT, 2011).

Para estes autores são apontados como critérios para a adoção de práticas de gerenciamento enxuto: (a) a garantia do comprometimento de todos os envolvidos no empreendimento; (b) a definição colaborada de etapas críticas e do monitoramento do projeto e da ação; (c) o uso de técnicas com vistas a melhorias incrementais (matrizes, verbalizações por meio de oficinas facilitadas, etc.); (d) a busca da eficiência utilizando a tecnologia; (e) a promoção da filosofia enxuta e o incentivo de seu uso e (f) a ênfase nas fases anteriores à construção.

Emmitt et al. (2011) constataram que a promoção da interação entre os envolvidos propagada pela cultura enxuta teve como reflexos para o gerenciamento do projeto a melhoria do fluxo de trabalho e menos desentendimentos na equipe. Verificou-se que, para a equipe de desenvolvimento de projeto, parece preferível melhorar o gerenciamento do trabalho coletivo 
do que organizá-lo por meio de roteiros ou fórmulas prontas, como por exemplo, softwares de administração. Esta observação é coerente com as dificuldades de aplicação do LDM e, por outro lado, também demonstra a ansiedade por parte dos agentes do processo em tornar o processo menos penoso e mais eficiente.

Para Tilley (2005) e El.Reifi; Emmitt (2011), a qualidade das soluções de projeto, sua capacidade de alcançar os requisitos do cliente e maximizar o valor dependem do nível de interação com o cliente e com os demais envolvidos no processo de projeto.

Por isso, como recurso para a consolidação das práticas de gerenciamento enxuto El.Reifi; Emmitt (2011), Tilley (2005) e Ghassemi; Becerik-Gerber (2011) destacam a importância da organização contratual e do uso de contratos relacionais, cuja característica é comprometer integralmente todas as partes num único instrumento de modo que se reforce o envolvimento e se construa confiança.

A intrincada relação entre os princípios do gerenciamento de projetos, o modelo produtivo de transformação e a teoria da produção impõem-se como um nevoeiro que dificulta a visibilidade de soluções que permitam melhorar o desempenho do processo de projeto e por isso mais estudos precisam ser desenvolvidos e o conhecimento divulgado (TILLEY, 2005; EL.REIFI; EMMITT, 2011).

\section{MÉTODO}

O método de pesquisa adotado para o desenvolvimento deste trabalho é classificado como qualitativo e foi realizado por meio de estudos de caso.

Os instrumentos utilizados para a coleta de dados foram a pesquisa bibliográfica, seguida da pesquisa de campo, utilizando-se para o levantamento de dados a aplicação de entrevistas semi-estruturadas e pesquisa documental dos escritórios estudados.

Para a elaboração do questionário semi-estruturado foram identificados como critérios orientadores para o aprimoramento do processo de projeto: o fluxo de informações, o gerenciamento de valor e o planejamento do processo.

Os seguintes critérios foram considerados na seleção das empresas:

- Ser responsáveis pela elaboração de projeto arquitetônico e complementares que atendessem ao mercado construtor goianiense de edifícios de multipavimentos.

- Atendessem clientes construtoras e/ou incorporadores de empreendimentos residenciais e comerciais.

- $\quad$ Ter como nicho de mercado a região metropolitana de Goiânia-GO e ser representativa no ramo de incorporação e construção neste cenário;

- $\quad$ Ter interesse na realização da pesquisa.

Mediante aos critérios definidos, três empresas foram contatadas, mas apenas duas responderam positivamente à participação da pesquisa. A terceira empresa não demonstrou interesse na participação da pesquisa. Os questionários semi-estruturados foram aplicados por meio de entrevistas em dois escritórios de projetos, um deles de arquitetura e outro de estruturas e ocorreram em dezembro de 2012 e janeiro de 2013, respectivamente.

Em cada empresa apenas um profissional foi entrevistado. No escritório de arquitetura foi entrevistado um dos dois diretores da empresa. Este diretor é um dos fundadores da empresa e, junto com seu sócio, controla todos os processos administrativos e de produção da empresa. Ele atua, sobretudo, no escritório, recebendo clientes e projetistas colaboradores para as 
reuniões de contrato, projeto e coordenação. No escritório de estruturas o participante da pesquisa foi um engenheiro civil pertencente ao quadro da empresa, responsável por coordenar uma das tipologias de projeto desenvolvidas pela: a de reforço de estruturas. Este engenheiro não atua em atividades administrativas do escritório, concentrando-se na produção.

O questionário, apresentado no Quadro 1, foi composto de três seções sendo a primeira dedicada à caracterização da empresa, a segunda com perguntas diretas e de múltipla escolha a respeito dos tópicos de fluxo de informações, o gerenciamento de valor e o planejamento do processo de projeto e a terceira com seis perguntas que objetivavam aprofundar no tema das três questões anteriores e verificar a coerência das respostas dadas.

\section{Quadro 1 - Questionário}

\begin{tabular}{|c|c|}
\hline \multicolumn{2}{|c|}{ Dados da Empresa } \\
\hline & po de atuação no mercado de edifícios $m$ \\
\hline \multicolumn{2}{|c|}{ 2. Nome do entrevistado e função no escritório. } \\
\hline \multicolumn{2}{|c|}{ 3. O escritório tem certificações? Quais? } \\
\hline \multicolumn{2}{|c|}{ 4. Número de projetos de edifícios multipavimentos entregues ou produzidos por ano. } \\
\hline \multicolumn{2}{|c|}{$\begin{array}{l}\text { 5. Breve descrição do funcionamento do escritório (Como um novo projeto entra na produção do escritório? } \\
\text { Quem são os responsáveis? Os auxiliares de projeto que atuam em sua elaboração constituem equipes? Há um } \\
\text { planejamento interno preliminar para a produção do projeto e programação de reuniões? Como é feita e por } \\
\text { que meios se dá a interação com os demais projetistas? Como são controlados os resultados dos projetos?) }\end{array}$} \\
\hline $\begin{array}{l}\text { Atendimento aos } \\
\text { requisitos do cliente }\end{array}$ & Descrição \\
\hline$\square \quad$ NÃO ATENDEU & Circunstâncias demandaram a reelaboração QUASE INTEGRAL do projeto \\
\hline NÃO ATENDEU & Circunstâncias demandaram a reelaboração de PARTES do projeto \\
\hline ATENDEU & Projeto executado integralmente com pequenas modificações \\
\hline ATENDEU & O projeto incorporou melhoramentos aos requisitos pedidos pelos clientes \\
\hline $\begin{array}{l}\text { Importância do fluxo } \\
\text { de informações }\end{array}$ & \\
\hline$\square \quad$ ALTO & $\begin{array}{l}\text { Uso de extranet e CURTOS intervalos de alimentação do sistema com informações } \\
\text { de projeto }\end{array}$ \\
\hline MÉDIO & $\begin{array}{l}\text { Uso de extranet e LONGOS intervalos de alimentação do sistema com informações } \\
\text { de projeto (mais de } 2 \text { semanas) }\end{array}$ \\
\hline BAIXO & Uso de CDs e e-mails para o registro e compartilhamento das informações de projeto \\
\hline NENHUM & $\begin{array}{l}\text { As informações de projeto e arquivos de desenho só são compartilhadas quando } \\
\text { solicitados pelos demais projetistas ou quando completos. }\end{array}$ \\
\hline $\begin{array}{l}\text { Deficiências no } \\
\text { planejamento do } \\
\text { processo de projeto }\end{array}$ & Descrição \\
\hline ALTA & $\begin{array}{l}\text { Não foi realizado planejamento do processo de projeto ou a definição formal de um } \\
\text { cronograma. }\end{array}$ \\
\hline MÉDIA & $\begin{array}{l}\text { Foi realizado o planejamento das etapas do projeto e definidos marcos no } \\
\text { cronograma para seu acompanhamento. }\end{array}$ \\
\hline BAIXA & $\begin{array}{l}\text { O planejamento foi realizado de forma colaborativa e foram definidos marcos no } \\
\text { cronograma para seu acompanhamento }\end{array}$ \\
\hline MÍNIMA & $\begin{array}{l}\text { O planejamento foi realizado de forma colaborativa, com o uso de marcos no } \\
\text { cronograma e indicadores para monitoramento do processo. }\end{array}$ \\
\hline \multicolumn{2}{|c|}{$\begin{array}{l}\text { 1. O quanto contribuiria para os resultados do projeto elaborado pelo escritório a realização de uma reunião } \\
\text { preliminar com os demais projetistas e outros agentes relevantes para a preparação do PLANEJAMENTO DO } \\
\text { PROCESSO DE PROJETO? }\end{array}$} \\
\hline $\begin{array}{l}\text { 2. O programa de nece } \\
\text { definição do progra } \\
\text { chave, construtores }\end{array}$ & $\begin{array}{l}\text { dades que subsidia os projetos elaborados pelo escritório é fornecido pelo cliente? A } \\
\text { de necessidades de forma colaborativa, reunindo cliente, projetistas, fornecedores- } \\
\text { usuários potenciais resultaria em melhorias para as soluções de projeto? Que }\end{array}$ \\
\hline
\end{tabular}


deficiências ou dificuldades seriam superadas com este tipo de procedimento?

3. A falta de compreensão completa dos requisitos do cliente antes do início do projeto geram alterações no projeto em suas fases mais avançadas, exigindo retrabalhos, o que significa mais dedicação dos trabalhadores e maior consumo de recursos e de tempo. Que soluções ou modificações na organização e no fluxo das informações necessárias para o projeto permitiriam que os requisitos do cliente fossem plenamente compreendidos antes da projetação?

4. Quais as vantagens e as dificuldades para o uso de ferramentas de planejamento do processo de projeto como cronogramas detalhados, marcos definidores de reuniões e entrega de produtos preliminares e o uso de indicadores de desempenho?

5. Que modificações nos contratos firmados entre projetistas e clientes contribuiriam para melhorar o comprometimento e o envolvimento dos diversos colaboradores de um projeto em seu planejamento?

6. Que medidas poderiam ser tomadas para minimizar ou até mesmo solucionar os problemas de interface (interoperabilidade) entre os projetos dos diversos escritórios e fornecedores envolvidos no processo?

Devido à pequena quantidade de entrevistas os resultados não puderam ser tabulados. A partir da comparação dos resultados procuramos identificar nos escritórios a existência de estratégias ou ações que visem o aprimoramento do processo, as dificuldades para a realização das melhorias e as contribuições que estas melhorias trariam para o processo de projeto.

\section{LEVANTAMENTOS DE DADOS E DISCUSSÃO DE RESULTADOS}

\subsection{Caracterização das empresas entrevistadas}

O escritório 1 é uma empresa de profissionais associados que presta serviços de consultoria e projetos estruturais com 40 anos no mercado. A empresa não possui certificações e produz uma média de 30 projetos de edifícios de multipavimentos por ano. Por ser uma empresa de pequeno porte é administrada por um único diretor-proprietário que concentra o controle administrativo e produtivo da empresa.

Partindo da diretoria do escritório existem distintas equipes de produção de projeto coordenadas por engenheiros civis. Cada equipe detém uma tipologia especial, com a qual pode trabalhar caso existam contratos que as demandem. As tipologias são alvenaria estrutural, obras industriais, reforços estruturais, estrutura metálica e concreto protendido.

O funcionamento do escritório não se dá por divisão de tarefas e sim por equipes e tipologia de projetos. Assim, não há um departamento exclusivo para lançamento de estruturas, outro para detalhamento de vigas, outro para detalhamento de pilares e etc. A diretoria do escritório acredita que a divisão por tarefas não contribui para o aprimoramento profissional do engenheiro e que a oportunidade de participar de um projeto do início é mais proveitosa, apesar de ser menos produtiva se comparada com o regime por tarefas.

O escritório tem por procedimento uma reunião de partida para a caracterização inicial do trabalho e faz uso de check-lists para o registro do programa inicial. Estas medidas têm por objetivo agilizar lançamento do partido estrutural e evitar retrabalhos. Cada equipe efetua seus registros e compartilha informações exclusivamente por e-mails. Ao longo do trabalho, o escritório aproveita as reuniões de coordenação dos empreendimentos e eventualmente as solicita para apresentar soluções ou demandas de novos dados.

O escritório 2 é uma empresa de profissionais associados que presta serviços de coordenação e elaboração de projetos de arquitetura com 10 anos no mercado. A empresa tem sistema de gestão da qualidade (ISO 9001/2008) certificado e uma produção média de 18 projetos de edifícios de multipavimentos por ano.

O escritório se organiza em três níveis: diretoria, coordenadores e profissionais responsáveis. São três coordenações responsáveis por tarefas distintas: (a) estudos de viabilidade técnica e 
projetos legais, (b) projetos executivos e detalhamentos e (c) coordenação de projetos complementares. Os profissionais responsáveis atuam dentro das coordenações no desenvolvimento das tarefas sob a orientação de profissionais arquitetos.

O sistema de gestão da qualidade orienta as práticas do escritório e cada departamento tem seu conjunto de procedimentos que se iniciam em reuniões de partida, seguem por instantes de análises críticas, validações internas, revisões e reuniões de entrega. Esta sequência é documentada, em cada departamento, por meio de documentos gráficos, check-lists, cronogramas e históricos que servem de registro de ações e de responsáveis, assim como de subsídio para o trabalho do departamento seguinte. O programa de necessidade no escritório 2 tem caráter dinâmico e é completado a cada fase do projeto.

Para as atividades internas do escritório 2 e para o compartilhamento de informações com agentes externos o e-mail é o recurso preferencial de comunicação, especialmente para a solicitação de informações.

\subsection{Análise e discussão de resultados}

Ambos os escritórios indicaram que atendem os requisitos do cliente, sendo que o escritório 1 tem de lidar com pequenas modificações nos projetos finalizados mais frequentemente que o escritório 2, que consegue incorporar melhoramentos aos requisitos pedidos pelos clientes. Esta diferença pode ser atribuída ao tipo de projeto. O projeto de arquitetura é anterior ao de estruturas e este, por ser complementar, sofre mais diretamente as influências dos determinantes executivos no canteiro de obras. Muitas vezes o edifício difere do projeto arquitetônico em função de modificações ocorridas nos projetos complementares.

Para o gerenciamento de valor são adotados check-lists e reuniões de partida nos dois escritórios. Práticas que podem ser consideradas alinhadas ao LDM. No escritório 2, reuniões de partida, com seus respectivos check-lists, são realizadas toda vez que um projeto avança de um departamento para outro, o que não acontece no escritório 1 por duas razões: o caráter complementar do projeto estrutural e por uma só equipe conduzir o projeto do início ao fim.

Para os dois escritórios a principal contribuição de ações que melhorem o atendimento dos requisitos do cliente é minimizar os retrabalhos.

A importância do fluxo de informações foi classificada como baixa pelos dois escritórios. Nas duas empresas o uso de CDs e e-mails para o registro e compartilhamento das informações de projeto constitui a forma mais comum de comunicação com outros projetistas e com o cliente. $\mathrm{O}$ uso de extranets acontece em ambos os escritórios quando o cliente faz uso deste recurso ou o exige, circunstância comum quando o cliente contrata um serviço de coordenação de projetos independente dos escritórios investigados.

A baixa importância do fluxo de informações se deve ao padrão de ferramentas de desenho e comunicação adotados pelo mercado. Ainda é restrito o uso de plataformas colaborativas para projeto de edifícios em Goiânia. Os softwares de desenho são essencialmente 2D e com baixa interoperabilidade. $\mathrm{O}$ fluxo de informações fragmentado marca o mercado goianiense de projetos de edifícios, situação que parece evoluir aos poucos sob a demanda dos clientes.

A principal contribuição de melhorias no fluxo de informações seria a redução da ocorrência de faltas ou de atrasos de dados que emperram os projetos. A coordenação de projetos parece ser uma ferramenta bem-vinda para iniciar esta melhoria por atuar simultaneamente no planejamento e na gestão da informação. $\mathrm{O}$ uso de ambientes colaborativos virtuais também é considerado positivo por dispensar a necessidade de que algum agente do processo tenha de ser o gerenciador de versões de arquivos. 
O fluxo de informação está mais intimamente ligado ao planejamento do processo de projeto do que com a organização do escritório, no entanto, uma correlação pode ser feita entre o fluxo de informações e a organização de cada escritório. O fluxo é eficaz e mais estanque no escritório 2 que é departamentalizado e possui sistema de gestão da qualidade, enquanto que no escritório 1, o fluxo é mais difuso e informal.

Assim como a importância do fluxo de informações, o planejamento do processo de projeto também é caracterizado pelo relacionamento com o cliente e apesar das divergências das respostas, as deficiências no planejamento existem e não são poucas. Os dois escritórios relatam que nos casos em que há uma coordenação efetiva estas deficiências são minimizadas e que marcos e cronogramas são estabelecidos para o cumprimento das metas. Por outro lado, quando a coordenação não existe o processo de projeto caminha conforme são repassadas as informações pelo cliente e pelos demais colaboradores.

Foi observado que o prazo, como quesito de planejamento, tem importância diferente para cada tipo de projeto. Para o escritório 1, o prazo é um gargalo e é o foco dos instrumentos contratuais, já para o escritório 2 o prazo de entrega tem de ser flexível por ser dependente de informações do cliente, que muitas vezes vacila em seus requisitos. No entanto, esta flexibilidade no escritório 2 não o exime de manter rigorosos cronogramas internos de trabalho, de modo a deixar os atrasos sob a responsabilidade do cliente.

A principal dificuldade verificada pelos escritórios para o uso de ferramentas de planejamento e controle é a falta de disciplina dos agentes participantes, sobretudo do cliente. Por isso, a possibilidade de se estruturar um bom planejamento parece ser condicionada pela noção e qualificação gerencial do cliente. O planejamento é mais eficaz se o cliente percebe ou sabe que planejar gera economia para o empreendimento como um todo. Todavia, se o cliente não monitora suas perdas e as origens delas, o planejamento pode parecer desnecessário.

Melhorias no planejamento contribuiriam com a compatibilização do projeto, que associada a um sistema de informações, permitiria que ela acontecesse a partir de visualizações em 3 dimensões, tornando-a mais eficaz. O planejamento também reduziria os prejuízos financeiros, fazendo com que os projetos tivessem menos interrupções e que permanecessem menos tempo dentro do escritório, comprometendo a captação de novos trabalhos. Por fim, contribuiria para reduzir a inércia do mercado profissional que é resistente a mudanças.

Novamente a coordenação de projetos e o ambiente colaborativo são recursos desejáveis. Neste trabalho não foram identificadas práticas enxutas consolidadas nos escritórios para se reduzir as deficiências do planejamento.

\section{CONSIDERAÇÕES FINAIS}

A relevância do estudo pode ser verificada no impacto causado pelas deficiências do processo na organização do trabalho dos escritórios. Pôde-se verificar que práticas coerentes com o LDM são insipientes nos escritórios investigados e que dos três tópicos definidos para a avaliação do gerenciamento do processo de projeto, a definição de ações de melhorias para o planejamento do processo é a mais promissora em função dos resultados que pode proporcionar sobre os outros dois tópicos, apesar de ser, dentre os três, o mais intrincado como também apontaram Emmitt et al. (2011) e El.Reifi, Emmitt (2011).

A visão de mercado e o indutor de melhorias no escritório 1 é a valorização da formação profissional e a qualidade do produto entregue. No escritório 2 a visão de mercado é a de melhoria do processo de trabalho aliada a um produto de qualidade e o indutor desta melhoria é a compreensão das tendências do mercado a partir do que acontece dentro e fora do país. Foi citado na entrevista feita no escritório 2 que a chegada de empreendedores estrangeiros no 
país tem sido um incentivo para que os escritórios avancem em seus processos sob o risco de perderem oportunidade de bons negócios e espaço no mercado de projetos.

As dificuldades e deficiências do planejamento do processo de projeto apontadas pelas entrevistas acentuam a ideia de uma origem cultural para o problema. No país, a prática do planejamento e do monitoramento ainda está em consolidação e por essa razão entraves desta ordem são para nós difíceis de serem superados. A resistência do setor profissional a mudanças e inovações relatada nas entrevistas demonstra a extensão desta dificuldade e já há algum tempo identificadas por Formoso e Tzortzopoulos (1999).

Os resultados deste estudo foram limitados pela pequena amostragem e também por não ser possível aprofundar a investigação sobre o nível de conhecimento dos escritórios sobre os princípios enxutos. Pesquisas futuras podem preencher estas lacunas e ainda buscar estabelecer qual é a real interferência dos clientes nas deficiências do planejamento do processo, assim como qual a contribuição do sistema de gestão da qualidade e da interação para a adoção de ferramentas de controle enxutas no processo de projeto.

\section{REFERÊNCIAS}

AMERICAN INSTITUTE OF ARCHITECTS (AIA) - California Council. Integrated Project Delivery: a working definition. 2007 . Disponível em: $<$ http://www.ipdca.net/images/Integrated\%20Pro ject\%20Delivery\%20Definition.pdf>. Acesso em: 13 jul. 2012.

EL.REIFI, M. H.; EMMITT, S. Lean Design Management: exploring perception and practice. In: CIB-096 CONFERENCE 2011, Vienna, Austria. Proceedings... CIB, 2011. p. 46-56.

EMMITT, S.; PASQUIRE, C.; MERTIA, B. Addressing the architect/contractor interface: lean design management perspective. In: CIB-096 CONFERENCE 2011, Vienna, Austria. Proceedings... CIB, 2011. p. 110-119.

FORMOSO, C. T. ; TZORTZOPOULOS, P. . Considerations on the application of lean construction principles to design management. In: INTERNATIONAL CONFERENCE ON LEAN CONSTRUCTION, 7., 1999, Berkeley, Califórnia. Proceedings ... Berkeley: University of Califórnia, 1999. p. 335-344

GHASSEMI, R.; BECERIK-GERBER, B. Transitioning to Integrated Project Delivery: Potential barriers and lessons learned. Lean Construction Journal, lean and integrated project delivery special issue, p. 32-52, 2011. Disponível em: <www.leanconstructionjournal.org $>$.

HANSEN, G. K.; OLSSON, N. O. E. Layered process-layered process: lean thinking and flexible solutions. Architectural Engineering and Design Management, London, v. 7, n. 2, p. 70-84, 2011. DOI: $10.1080 / 17452007.2011 .582331$

KOSKELA, L.; BALLARD, G.; TANHUANPAA, V-P; Toward lean design management. In: $5^{\text {th }}$ Annual Conference of the International Group for Lean Construction, 1997, Gold Coast. Proceedings... Disponível em $<$ http://iglc.net/?page_id=6>

SMITH, R.E.; MOSSMAN,A.; EMMITT, S. Editorial: lean and integrated project delivery. Lean Construction Journal, lean and integrated project delivery special issue, p. 01-16, 2011. Disponível em: <www.leanconstructionjournal.org $>$.

THYSSEN, M. H.; EMMITT, E.; BONKE, S.; CHRISTOFFERSEN, A. K. Facilitating Client Value Creation en the Conception Design Phase of Construction Project: a workshop approach. Architectural, Engineering and Design Management, v. 6, n. 1, p. 18-30, 2010.

TILLEY, P. A. Lean Design Management: a new paradigm for managing the design and documentation process to improve quality. In: $13^{\text {th }}$ Annual Conference of the International Group for Lean Construction, 2005, Sydney. Proceedings... Disponível em $<$ http://iglc.net/?page_id=6 >. 
TRIBELSKY,E.; SACKS, R. Measuring information flow in the detail design of construction projects. Research in Engineering Design. n. 21, p. 189-206, 2010, DOI: 10.1007/s00163-009-0084-3. 